\title{
Mon patient est-il capable de discernement?
}

\author{
Ursina Pally Hofmann \\ Dr iur., avocate, secrétaire générale et responsable du Service juridique
}

\section{Capacité de discernement ou absence de celle-ci}

Une personne est capable de discernement dès lors qu'elle est en mesure d'agir raisonnablement. Cela signifie d'une part qu'elle est capable de se forger une volonté propre, c.-à-d. qu'elle est en mesure de comprendre et d'évaluer le sens, l'utilité et les conséquences d'un comportement donné, et d'autre part qu'elle est capable d'agir sur la base de cette opinion propre [1]. Lorsque tel est le cas, le patient a le droit de prendre une décision, même si elle n'est pas rationnelle du point de vue médical. Pour qu'il soit considéré comme capable de discernement, il ne faut donc pas que le patient agisse de manière rationnelle, d'un point de vue médical et objectif, mais qu'il soit en mesure de le faire [2]. Par ailleurs, la notion de capacité de discernement doit s'entendre pour un temps donné et un sujet donné, ce qui signifie qu'une personne est capable de discernement à un moment précis, pour une décision précise. Plus la situation est complexe et plus le niveau de discernement requis est élevé.

A l'inverse, pour qu'un patient soit considéré comme incapable de discernement, il faut que ses aptitudes mentales soient considérablement diminuées. C'est pourquoi le législateur suisse part du principe que toute personne est capable de discernement [3]. Il découle de cette présomption légale que le médecin peut, lui aussi, partir du principe que son patient est capable de discernement. Il n'est donc pas nécessaire de procéder à des vérifications particulières ni de documenter ses constatations. En raison de cette présomption, le médecin ne supporte pas le fardeau de la preuve et n'a donc pas à confirmer ou à prouver l'existence de la capacité de discernement.

\section{Nécessité de l'évaluation}

La loi mentionne toutefois des états de faiblesse dans lesquels on ne peut plus présumer la capacité de discernement du patient. Outre le jeune âge, la maladie psychique ou le handicap mental, des états d'ébriété ou d'intoxication de tout ordre peuvent priver une personne de sa capacité de discernement. Dès lors qu'un patient présente un état de ce type, le médecin doit obligatoirement se po- ser la question de la capacité de discernement et ne peut plus se reposer sur la présomption légale [4].

Tous les états de faiblesse n'ont pas pour effet d'entraver la capacité de discernement [5], raison pour laquelle on procédera à une évaluation. La loi ne prescrit pas de modalité particulière pour celle-ci. Dans un premier temps, une vérification informelle est suffisante. Une documentation sommaire suffit, tant que le médecin considère que la capacité de discernement est donnée et qu'une vérification approfondie n'apparaît pas nécessaire. Pour aider les médecins lors d'évaluations approfondies, l'Académie suisse des sciences médicales a édicté la directive «La capacité de discernement dans la pratique médicale» [6]. Outre le renvoi à la réglementation, cette publication aborde diverses situations tirées de la pratique médicale quotidienne, notamment le traitement d'enfants et d'adolescents, de patients présentant des troubles psychiques ou de traitements d'urgence.

L'exemple des patients mineurs illustre bien qu'il n'existe pas, même pour les états de faiblesse évoqués dans la loi et les groupes de patients mentionnés dans la directive, de règle universelle sur la capacité de discernement. On peut ainsi facilement concevoir qu'à 17 ans, une jeune patiente soit tout autant capable de se déterminer en vue d'une intervention chirurgicale que si elle avait atteint sa majorité officielle. De même, il va de soi qu'on ne pourra jamais considérer un nourrisson comme capable de discernement. En l'absence de limite d'âge clairement définie à partir de laquelle un patient mineur serait réputé capable de discernement pour certaines interventions, il incombe au médecin de déterminer ce qu'il en est, et de consigner ses conclusions par écrit. En fonction de son appréciation, il choisira d'impliquer de telle ou telle manière les parents dans les décisions thérapeutiques. Nous reviendrons sur ce point plus loin [7].

Mais ce n'est pas uniquement chez les patients mineurs qu'il importe d'adopter une approche différenciée. L'état psychique d'un même patient peut évoluer dans le temps, et chaque situation exige des degrés de discernement différents.

Indépendamment d'indices révélant une éventuelle incapacité de discernement chez un patient, certaines situations médicales exigent en soi que l'on procède à une évaluation et que l'on consigne les résultats. C'est 
par exemple le cas pour la stérilisation ou le don d'organes du vivant du donneur [8]. La jurisprudence exige également une documentation particulièrement méticuleuse dans le cadre de l'aide au suicide [9].

Dès qu'il peut exister un doute sur la capacité de discernement du patient, en raison d'un état de faiblesse, il y a un renversement du fardeau de la preuve. En cas de litige, le médecin devra démontrer qu'il a identifié l'état mental particulier de son patient, mené le travail de réflexion adéquat et procédé à l'évaluation qui s'imposait. Il veillera par ailleurs à consigner ses conclusions par écrit et à les motiver. La législation n'exige pas explicitement d'établir une documentation de ce type, mais à défaut, il va de soi que le médecin aura beaucoup de peine à prouver qu'il a agi correctement.

\section{Devoir d'information et capacité de discernement}

Le médecin a un devoir d'information à l'égard de son patient dès lors qu'il est capable de discernement. En revanche, un patient incapable de discernement ne peut pas être informé de façon adéquate, raison pour laquelle il ne peut pas consentir valablement à un acte médical. Celui-ci serait dès lors illicite et pourrait donner lieu à des poursuites pénales, à une action en responsabilité civile, ou encore à une procédure disciplinaire ou déontologique. Contrairement à la capacité de discernement, l'information du patient n'est pas présumée. Le médecin est tenu d'informer son patient avant chaque intervention ou chaque traitement. De même, il doit obtenir son consentement et documenter l'information au patient. La question du discernement ne doit en revanche être abordée que lorsque les circonstances le requièrent. On opérera dès lors une distinction entre la capacité de discernement et le devoir d'information.

\section{Que faire lorsque le patient est incapable de discernement?}

Le patient n'est pas, dans ce cas, la personne à qui l'information doit être adressée. Pour les personnes majeures (>18 ans [10]) incapables de discernement, la législation prévoit une liste, en cascade, de personnes habilitées à les représenter. Cette liste commence par les personnes désignées dans les directives anticipées ou dans un mandat pour cause d'inaptitude, passe ensuite au curateur chargé de représenter le patient dans le domaine médical, puis au conjoint ou au partenaire enregistré, dès lors qu'il fait ménage commun avec la personne incapable de discernement et lui fournit une assistance personnelle régulière, et se clôt par les frères et sœurs lui fournissant une assistance personnelle régulière. $\mathrm{Si}$ plusieurs représentants disposant des mêmes droits ne parviennent pas à un accord, ou si les intérêts du patient sont menacés de quelque manière que ce soit, on pourra faire appel à l'autorité de protection de l'adulte [11]. Le médecin agissant de bonne foi pourra toutefois assumer que les représentants agissent de concert [12].

Les patients mineurs incapables de discernement sont représentés par leurs parents, par un tuteur ou par un curateur chargé de la représentation pour les questions d'ordre médical. Faute d'éléments indiquant le contraire, on peut partir du principe que chaque parent agit en accord avec l'autre [13].

Le représentant, dans la mesure où il est lui-même capable de discernement, est le destinataire de l'information au patient. On se fondera sur le plan de traitement établi par le médecin [14]. Le représentant ne peut agir que de façon conforme à la volonté présumée du patient, ou à une volonté exprimée alors qu'il disposait de sa capacité de discernement. La volonté du représentant n'est pas prise en compte si elle ne correspond pas à la volonté du patient.

S'il n'est pas possible d'établir la volonté du patient, la personne habilitée à le représenter devra agir dans son intérêt objectif [15]. La même chose vaut pour le médecin, lorsqu'il est amené à décider en urgence du traitement à administrer sans qu'il ait connaissance de la volonté présumée du patient et en l'absence de représentant [16] On impliquera dans la mesure du possible le patient incapable de discernement dans le processus de décision [17]. Cela vaut pour les patients tant adultes que mineurs. $\mathrm{Au}$ cas où il se serait à tort adressé à un représentant alors que le patient avait la capacité de discernement, le médecin peut se voir reprocher d'avoir violé le secret professionnel et le droit de la protection des données [18]. Même pour les mineurs, on n'impliquera pas systématiquement les parents ou le tuteur dans les entretiens avec le patient et dans les décisions relatives au traitement. Lorsqu'un mineur peut être considéré comme capable de discernement, on veillera toujours à recueillir son assentiment au préalable. Dans les cas où les parents ou le tuteur ont été impliqués dans le traitement, avec le consentement du mineur capable de discernement, c'est néanmoins lui qui est considéré comme le destinataire de l'information au patient, et lui seul peut consentir au traitement ou le refuser.

\section{Finalité}

Le respect des règles sur la capacité de discernement, l'information au patient et son consentement permet de garantir le droit de ce dernier à l'autodétermination. De plus, le médecin qui se tient à ces règles sera en meilleure position pour se défendre contre d'éventuels reproches injustifiés qui lui seraient faits dans le cadre d'une procédure judiciaire. 